\title{
Direct detection and collider interplay in decoding the nature of Dark Matter
}

\author{
Alexander Belyaev* \\ School of Physics and Astronomy, University of Southampton, Southampton SO17 1BJ, UK \\ E-mail: a.belyaev@soton.ac.uk
}

Determination of the nature of Dark Matter (DM) is one of the most fundamental problems of particle physics and cosmology. If DM is light enough and interacts with Standard Model particles directly or via some mediators with a strength beyond the gravitational one, it can be directly produced at the Large Hadron Collider or future particle accelerators. The typical signature from DM produced in particles collisions is missing transverse energy, MET, due to the fact that they escape undetected from the experimental apparatus. We present study of the complete set of dimension 5 and 6 effective operators with scalar, fermion and vector DM and explore possibility to distinguish these operators and characterise the spin of DM. We have found that, depending on the spin of the DM, the DM parts of the effective operators lead to a different energy dependence of the cross-sections and to different distributions of the invariant mass of the DM pair, and consequently to different MET distributions. We have found that at the LHC with high luminosity, certain classes of EFT operators can be distinguished from each other and, through this, it is possible to characterise the spin of DM in some cases. This study can be directly applied beyond the EFT paradigm, as we demonstrate for two cases - Supersymmetry and inert two Higgs doublet model (i2HDM). We find limits using LHC mono-jet data, spin independent and spin dependent direct searches, relic density and $\mathrm{CMB}$, and show an important role of the interplay between high and low energy data in exploring DM and identification its nature. We also highlight prospects of new signature from DM theories such as disappearing charge tracks which are characteristic for wide class of DM theories and demonstrate the LHC potential to explore them using examples with of i2HDM and Minimal Spin-one Isotriplet Dark Matter models. We show that collider and non-collider DM searches have a unique power to probe the nature of Dark Matter using examples of several appealing DM theories.

2nd World Summit: Exploring the Dark Side of the Universe

25-29 June, 2018 - EDSU2018

University of Antilles, Pointe-à-Pitre, Guadeloupe, France

${ }^{*}$ Speaker. 


\section{Introduction}

Understanding the nature of Dark Matter (DM) is one of the greatest puzzles of modern particle physics and cosmology. Although overwhelming observational evidences from galactic to cosmological scales point to the existence of DM [1, 2, 3], after decades of experimental effort only its gravitational interaction has been experimentally confirmed. Currently, no information is available on the DM properties, such as its spin, mass, interactions other than gravitational, symmetry responsible for its stability, number of states associated to it, and possible particles that would mediate the interactions between DM and the standard model (SM) particles.

If DM is light enough and interacts with SM particles directly or via some mediators with a strength beyond the gravitational one, its elusive nature can be detected or constrained in different ways: a) from direct production at colliders, resulting in a signature exhibiting an observed SM object, such as jet, Higgs, $Z$, or photon, that recoils against the missing energy from the DM pair [4, 5, 6, 7]; b) via the relic density constraint obtained through the observations of cosmic microwave background (CMB) anisotropies, such as those of WMAP and PLANCK collaborations [8, 1];c) from DM direct detection (DD) experiments, which are sensitive to elastic spin independent (SI) or spin dependent (SD) DM scattering off nuclei $[9,10,11,12]$; d) from DM indirect detection searches, that look for SM particles produced in the decay or annihilation of DM present in the cosmos, both with high energies observables (gamma-rays, neutrinos, charge cosmic rays) produced in the local Universe $[13,14,15,16,17,18]$, and by studying the effects of energy produced by DM annihilation in the early universe on the properties of the CMB spectrum [19, 20, 1].

It is clear that decoding of the nature of
DM requires the respective signal at least in one of the search experiment. We do not have one. However even without having this signal at the moment we can already conclude on what kind of DM models are excluded already. Moreover, by exploring different signatures of one particular model, their correlation and interplay we can prepare ourselves to discovery of DM and their identification.

\section{Contact interactions}

In Table 1 we have summarised a minimal set of independent dimension-5 and dimension6 operators for complex scalar, Dirac fermion and complex vector DM coupling to quarks and gluons, adopting the widely used notations of $[21,22,23]$. These operators provide monojetsignature, the shapes of $\boldsymbol{E}_{\mathrm{T}}^{\text {miss }}$ distributions for which is presented in in Fig. 1 from Ref. [23] for DM mass of $10 \mathrm{GeV}$. One can observe a big difference in $\boldsymbol{E}_{\mathrm{T}}^{\text {miss }}$ shapes of the groups of the operators, primarily split into groups of operators with scalar, femion and vector DM. The origin of the different $\boldsymbol{E}_{\mathrm{T}}^{\text {miss }}$ shapes from different operators can be related to a combination of effects. First, for a fixed Lorentz structure of the SM part of the EFT operators, the same invariant mass distribution of the DM pair, $M_{\text {inv }}(D M, D M)$, uniquely defines the shape of the $\boldsymbol{E}_{\mathrm{T}}^{\text {miss }}$ distribution. Moreover, with the increase of $M_{\mathrm{inv}}(D M, D M)$, the $\boldsymbol{E}_{\mathrm{T}}^{\text {miss }}$ shape falls less and less steeply (again, for a given SM component of the EFT operator).

It was found in Ref. [23] that the reason why the bigger invariant mass of DM is correlated with flatter $\boldsymbol{E}_{\mathrm{T}}^{\text {miss }}$ behaviour is simply related to phase space and parton density effects: when $M_{\text {inv }}(D M, D M)$ is small, the radiation of a high $P_{T}$ jet will "cost" a large relative shift in $x$, the transferred momentum of the parton, leading to a rapidly falling $\boldsymbol{E}_{\mathrm{T}}^{\text {miss }}$ distribution; on the contrary, when $M_{\mathrm{inv}}(D M, D M)$ is large, the radiation of a high $P_{T}$ jet will "cost" a small relative shift in $x$, which will lead to a more 
slowly falling $\boldsymbol{E}_{\mathrm{T}}^{\text {miss }}$ distribution in comparison to the first case.

\begin{tabular}{|c|c|}
\hline \multicolumn{2}{|l|}{ Complex Scalar DM } \\
\hline $\begin{array}{l}\frac{g_{*}^{2}}{\Lambda} \phi^{\dagger} \phi \bar{q} q \\
\frac{g_{*}^{2}}{\Lambda} \phi^{\dagger} \phi \bar{q} i \gamma^{5} q \\
\frac{g_{*}^{2}}{\Lambda^{2}} \phi^{\dagger} i \overleftrightarrow{\partial_{\mu}} \phi \bar{q} \gamma^{\mu} q \\
\frac{g_{*}^{2}}{\Lambda^{2}} \phi^{\dagger} i \overleftrightarrow{\partial_{\mu}} \phi \bar{q} \gamma^{\mu} \gamma^{5} q\end{array}$ & $\begin{array}{l}{[C 1]} \\
{[C 2]} \\
{[C 3]} \\
{[C 4]}\end{array}$ \\
\hline 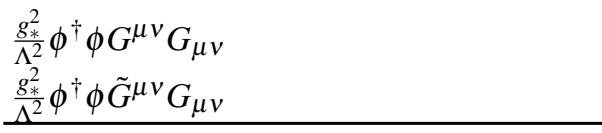 & \\
\hline \multicolumn{2}{|l|}{ Dirac Fermion DM } \\
\hline $\begin{array}{l}\frac{g_{*}^{2}}{\Lambda^{2}} \bar{\chi} \chi \bar{q} q \\
\frac{g_{*}^{2}}{\Lambda^{2}} \bar{\chi} i \gamma^{5} \chi \bar{q} q \\
\frac{g_{*}^{2}}{\Lambda^{2}} \bar{\chi} \chi \bar{q} i \gamma^{5} q \\
\frac{g_{*}^{2}}{\Lambda^{2}} \bar{\chi} \gamma^{5} \chi \bar{q} \gamma^{5} q \\
\frac{g_{*}^{2}}{\Lambda^{2}} \bar{\chi} \gamma^{\mu} \chi \bar{q} \gamma_{\mu} q \\
\frac{g_{*}^{2}}{\Lambda^{2}} \bar{\chi} \gamma^{\mu} \gamma^{5} \chi \bar{q} \gamma_{\mu} q \\
\frac{g_{*}^{2}}{\Lambda^{2}} \bar{\chi} \gamma^{\mu} \chi \bar{q} \gamma_{\mu} \gamma^{5} q \\
\frac{g_{*}^{2}}{\Lambda^{2}} \bar{\chi} \gamma^{\mu} \gamma^{5} \chi \bar{q} \gamma_{\mu} \gamma^{5} q \\
\frac{g_{*}^{2}}{\Lambda^{2}} \bar{\chi} \sigma^{\mu v} \chi \bar{q} \sigma_{\mu v} q \\
\frac{g_{*}}{\Lambda^{2}} \bar{\chi} \sigma^{\mu v} i \gamma^{5} \chi \bar{q} \sigma_{\mu \nu} q\end{array}$ & $\begin{array}{l}{[\mathrm{D} 1]} \\
{[\mathrm{D} 2]} \\
{[\mathrm{D} 3]} \\
{[\mathrm{D} 4]} \\
{[\mathrm{D} 5]} \\
{[\mathrm{D} 6]} \\
{[\mathrm{D} 7]} \\
{[\mathrm{D} 8]} \\
{[\mathrm{D} 9]} \\
{[\mathrm{D} 10]}\end{array}$ \\
\hline \multicolumn{2}{|l|}{ Complex Vector DM } \\
\hline 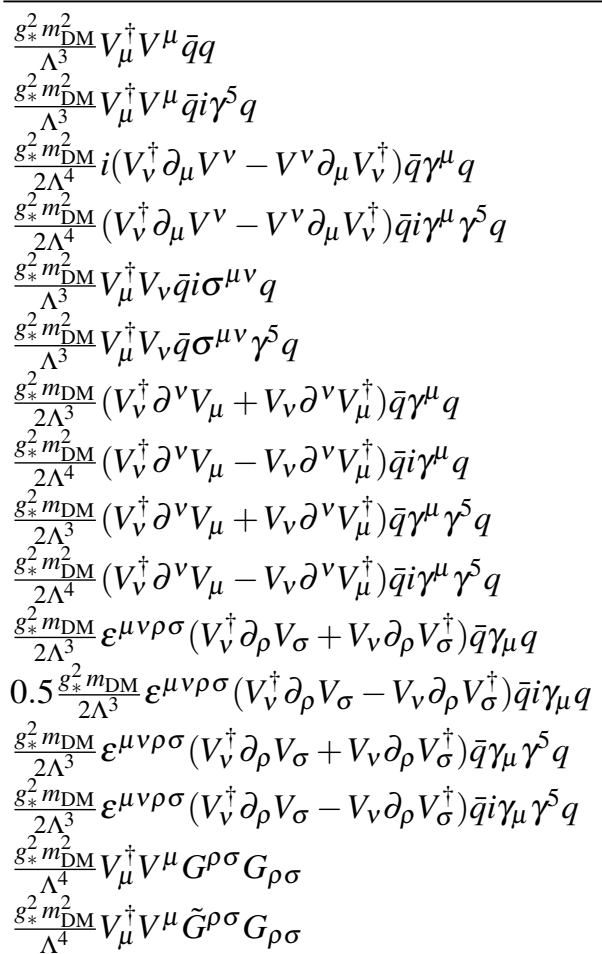 & $\begin{array}{l}{[\mathrm{V} 1]} \\
{[\mathrm{V} 2]} \\
{[\mathrm{V} 3]} \\
{[\mathrm{V} 4]} \\
{[\mathrm{V} 5]} \\
{[\mathrm{V} 6]} \\
{[\mathrm{V} 7 \mathrm{P}]} \\
{[\mathrm{V} 7 \mathrm{M}]} \\
{[\mathrm{V} 8 \mathrm{P}]} \\
{[\mathrm{V} 8 \mathrm{M}]} \\
{[\mathrm{V} 9 \mathrm{P}]} \\
{[\mathrm{V} 9 \mathrm{M}]} \\
{[\mathrm{V} 10 \mathrm{P}]} \\
{[\mathrm{V} 10 \mathrm{M}}\end{array}$ \\
\hline
\end{tabular}

Table 1: Minimal basis of operators of dimension six or less involving only complex scalar DM $(\phi)$, Dirac fermion DM $(\chi)$ or complex vector DM $\left(V^{\mu}\right)$ interacting with SM quarks $(q)$ or gluons. Here we denote the field strength tensor of the gluons as $G^{\mu v}$ and its dual as $\tilde{G}^{\mu \nu}$.

Since different oprators have differentt energy behaviour and respective different invariant mass distributions: typically softer for majority operators with scalar DM, intermediate for fermion DM and the hardest for vector DM and because of relation of $M_{\mathrm{inv}}(D M, D M)$ and $\boldsymbol{E}_{\mathrm{T}}^{\mathrm{miss}}$ slope one can distinguish many operators and related underlying theories between ear other by the shape of the $\boldsymbol{E}_{\mathrm{T}}^{\text {miss }}$ signal: C1C2,C5-C6,D9-D10,V1-V2,V3-V4,V5-V6 and V11-12 pairs among each other [23](that the signal is observed).

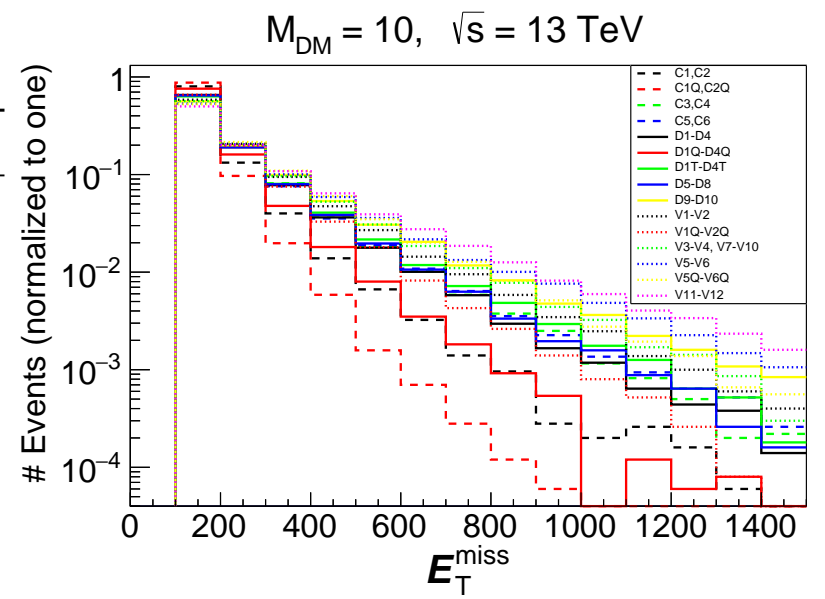

Figure 1: $\boldsymbol{E}_{\mathrm{T}}^{\mathrm{miss}}$ parton level distributions for a representative subset of the EFT operators from Table 1 for $13 \mathrm{TeV}$ LHC energy and $M_{\mathrm{DM}}=10 \mathrm{GeV}$.

Non-collider DM searches play an important complementary role in probing DM parameter space. As an example in Fig. 2 (top) we present the non-collider constraints for the operators D2, which exhibit pseudo-scalar interactions of fermion Dirac DM with quarks. 


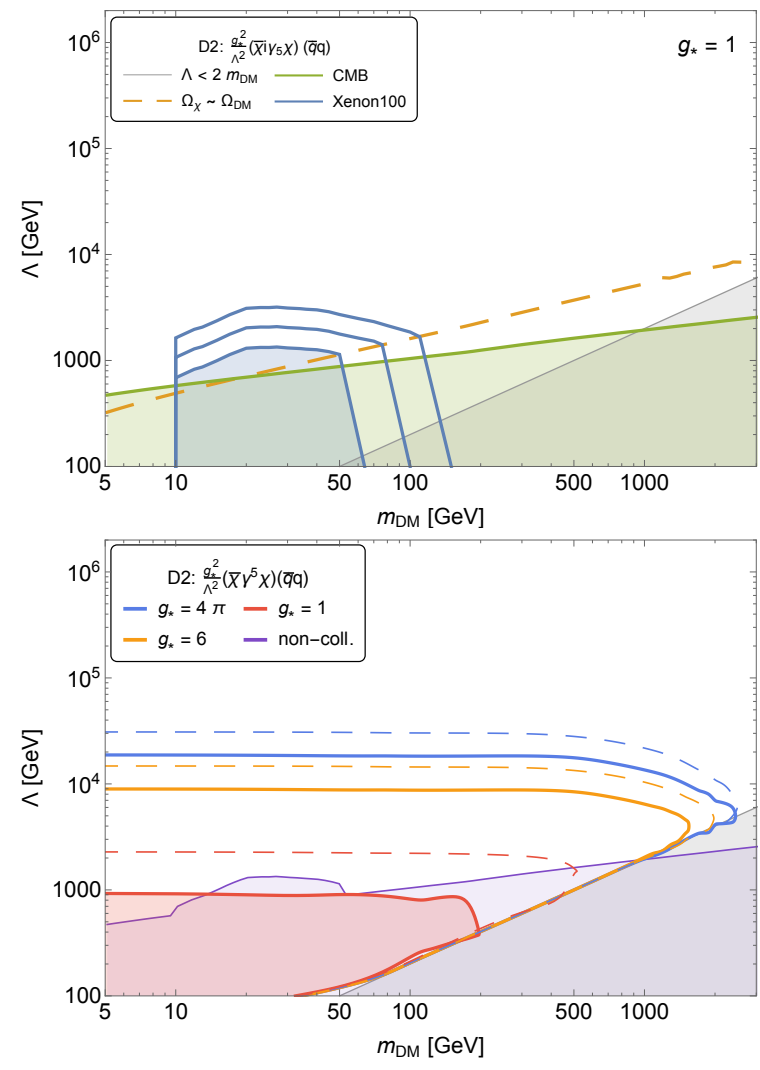

Figure 2: Top: Non-collider constraints on D2 operator with fermion DM: (i) SI DM DD searches (shaded blue region below the lowest blue contour), (ii) constraints from relic density (above the yellow dashed line), (iii) constraints from the CMB ( shaded green area) and (iv) constraints from the validity of the EFT $\left(\Lambda>2 m_{D M}\right)$. Bottom: LHC monojet constraints on D2 EFT operator. The area inside the red, orange and blue solid curves is excluded by current LHC data at 95\% CL for $g_{\star}=1$, 6 and $4 \pi$, respectively. The projected LHC limits for $300 \mathrm{fb}^{-1}$ are indicated by dashed thin lines. The combined exclusion regions from $\mathrm{CMB}$ and DM DD searches for $g_{\star}=1$ are given by the lightpurple area. See details and complete set of plots in Ref [24].

One can see that even for momentumsuppressed operator D2 (because of its pseudoscalar nature) DM DD constraints from Xenon[25] play an important role which is comparable to collider constraints, presented in Fig. 2 (bottom). It is important to stress that both LHC and DM DD searches set an upper limit on value of $\Lambda$. The LHC limit is of the order of $1 \mathrm{TeV}$ for present LHC data while DM DD searches the limit strongly depend on the operator. For example for non-suppressed operators conserving parity the limit on $\Lambda$ is about 3 orders of magnitude above the LHC one. On the other hand LHC limit is beyond DM DD searches for operators with suppressed elastic scattering cross sections on the nuclei (C2,C4,C6,D2,D3,D4,D610,V2,V4-V10). Moreover for operators with pseudo-vector currents which have suppressed DM DD rates, one should take into account effect of their running from $\mathrm{TeV}$ energy scale at the LHC down to low energy scale at DM DD experiments, due to which an operator acquire non-negligible vector component [26, 27, 28].

\section{Beyond EFT}

The analysis of $\boldsymbol{E}_{\mathrm{T}}^{\text {miss }}$ shape presented here can be applied to different scenarios, beyond the EFT approach in general, where the DM mediator is not produced on-the-mass-shell, such as the case of t-channel mediator or mediators with mass below $2 M_{D M}$, where the $M_{\text {inv }}(D M, D M)$ is not fixed. This case covers a wide range of theories. As an example in Fig. 3(top) the normalised shape for $\boldsymbol{E}_{\mathrm{T}}^{\text {miss }}$ distribution from $p p \rightarrow \chi_{1}^{+} \chi_{1}^{-} / \chi_{1}^{ \pm} \chi_{1}^{0} \rightarrow$ $\chi_{1}^{0} \chi_{1}^{0}+$ softletons/jets Minimal Supersymmetric Model(MSSM) signal and its dominant irreducible background $Z+j e t \rightarrow v \bar{v}+$ jet $(Z j)$ is presented for LHC@13TeV [29]. In the Fig. 3(bottom) we present $\boldsymbol{E}_{\mathrm{T}}^{\text {miss }}$ from $h_{1} h_{2} j$ inert two Higgs doublet model (i2HDM) signal alongside the estimated (by CMS) experimental background for $\sqrt{s}=13 \mathrm{TeV}$. An important feature of the signal versus background shapes in these completely different theory cases is that the the background falls more rapidly with $\boldsymbol{E}_{\mathrm{T}}^{\mathrm{miss}}$, and the difference in the slope with respect to the signal is bigger for higher DM mass. This behaviour has the same explana- 
tion as for EFT study case above - it is related to the bigger invariant mass of the invisible system for the signal $-M_{\text {inv }}(D M, D M)$ than for the background $-M_{Z}$. This feature provides a very important way to increase signalto-background ratio (S/B) (which is typically below $1 \%$ for low $\boldsymbol{E}_{\mathrm{T}}^{\text {miss }}$ cuts) by increasing the value of $\boldsymbol{E}_{\mathrm{T}}^{\text {miss }}$ or by performing the signalbackground shape analysis [30].
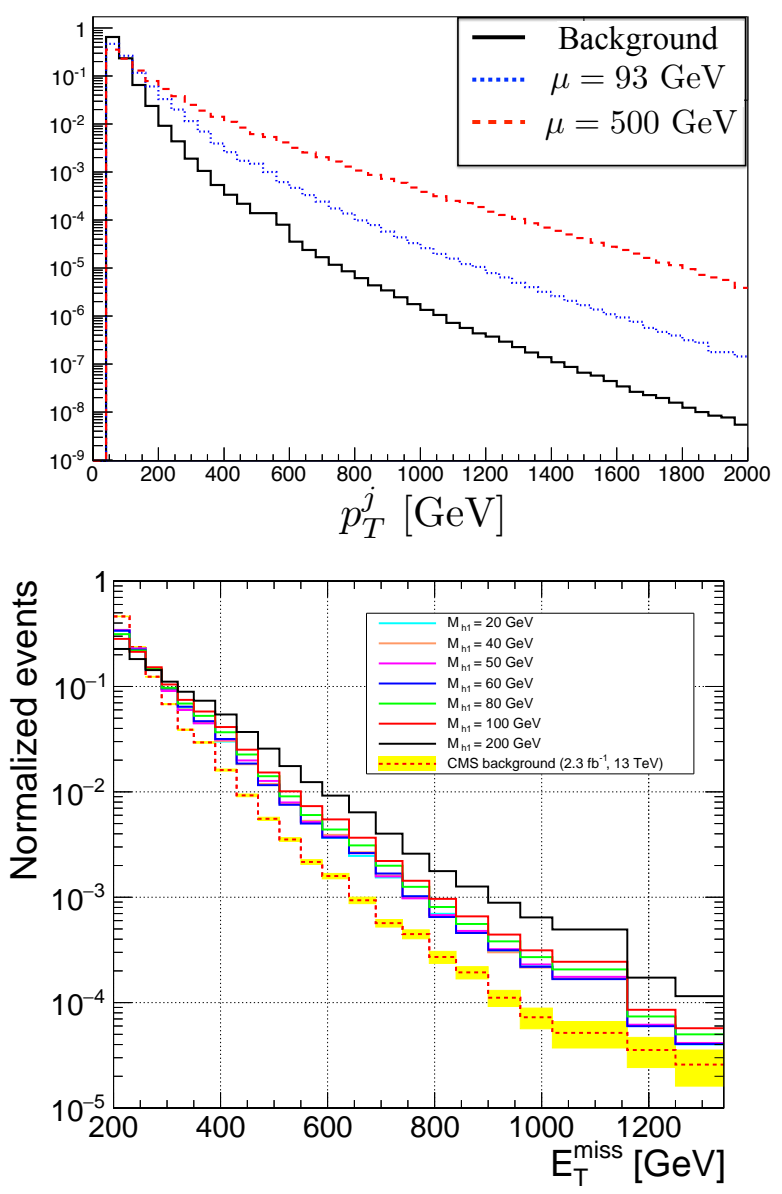

Figure 3: Top: Signal (dotted blue and dashed red) and $\mathrm{Zj}$ background (solid black) parton-level $p_{T}^{j}$ distributions for the $13 \mathrm{TeV}$ LHC for the NSUSY scenario: normalised signal and $Z j$ background distributions. See details in Ref [29]. Bottom: $\boldsymbol{E}_{\mathrm{T}}^{\text {miss }}$ from $h_{1} h_{2} j$ i2HDM signal vs background for $\sqrt{s}=$ $13 \mathrm{TeV}$. See details in [30]

The role of non-collider DM searches is also crucial in case of these two com- plete and consistent models. And an example in Fig. 4 we present the projected LHC reach for MSSM monojet signal in the $\Delta M=$ $m_{\chi_{1}^{+}}-m_{c h i_{1}^{0}}, M_{D M}=m_{c h i_{1}^{0}}$ parameter space together with LUX anx Xenon1T DM DD exclusion Ref [29]. One can see that LHC would be able cover neutralino DM mass only below 250 $\mathrm{GeV}$ (with the assumption that $\mathrm{S} / \mathrm{B}$ of the order of $3 \%$ will be under control) even with $3 \mathrm{ab}^{-1}$ total integrated luminocity. It is worth to stress though that LHC will cover the region inaccessible by Xenon1T in small $\Delta M$ region, while Xenon $1 \mathrm{~T}$ is able to cover $m_{D M}$ well beyond the LHC reach for $\Delta M>3-5 \mathrm{GeV}$, demonstrating a very important complementarity of DM DD to collider searches of DM. In case of i2HDM model collider sensitivity with mono-jet signature is even more limited because of the lower production rates of the scalar DM, $h_{1}$, or its inert partners $\left(h_{2}\right.$ and $\left.h^{+}\right)$and expected LHC reach is below $100 \mathrm{GeV}$ for $M_{h 1}$.

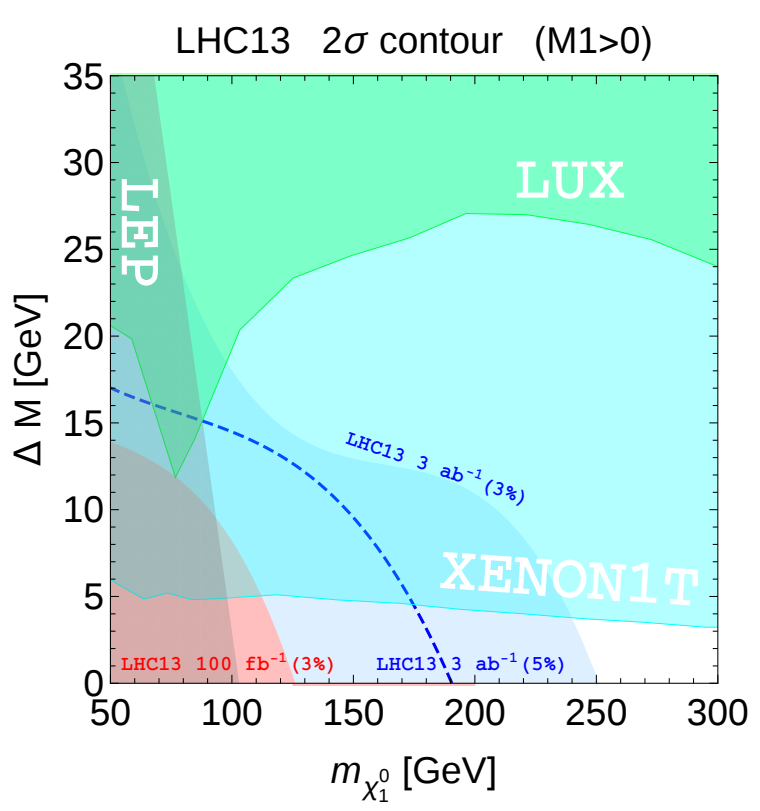

Figure 4: Exclusion contour lines for the $13 \mathrm{TeV}$ LHC at the end of the LHC Run2 (light red region) and of the HL-LHC (light blue region). The region excluded by LUX and Xenon1T are also shown, together with the LEP limit. See details in Ref [29]. 


\section{Beyond mono-X signature}

While mono- $X$ (with $X$ being jet, $\gamma, Z, H, t$ etc.) DM signatures at colliders are the most general ones, their rates is typically very low (usually at the percent level or even lower). Besides several others interesting but modelspecific DM signature studies one should stress one signature which can be also considered as quite generic one. In case when DM, $D^{0}$, is embedded into electroweak multiplet and its mass split from the charged odd particle(s), $D^{+}$, is generated only radiatively (preserving gauge invariance), the one can find that the value of this mass split is of the order of $0.2 \mathrm{GeV}$. In this case $D^{+}$has a very small width and respectively large life-time driven by its dominant decay to $\mathrm{DM}$ and pion: $D^{+} \rightarrow D^{0} \pi^{0}$ which makes $D^{+}$long lived particles (LLP). Production of $D^{+}$in pairs or in association with DM leads then to the typical signature from charged LLP: disappearing charged tack (DCT) as soon as the track from LLP is long enough (from few $\mathrm{cm}$ to a meeter). In case of such signature the S/B ratio is much higher than in case of mono-jet signal and therefore, substantially bigger DM masses can be probed with charged LLPs from DM sector [31, 32, 33]. As an example, we would like to present here results for the minimal vector triplet DM $\left(V^{0}\right)$ model [33] which predicts the right amount of $\mathrm{DM}$ for $M_{D M}$ in the 3-4 TeV range depending on DM coupling tot he Higgs boson. In Fig. 5(top) we present results for spin-independent crosssection for $V^{0}$-nucleon elastic scattering as a function of $M_{V}$ and for representative values of $a$. It is very important to note that Xenon1T experiment excludes DM mass above $4 \mathrm{TeV}$, while from Fig. 5(bottom) one can see that $100 \mathrm{TeV}$ collider will be able to exclude DM mass below $4 \mathrm{TeV}$, thus allowing to probe the entire parameter space of the model.
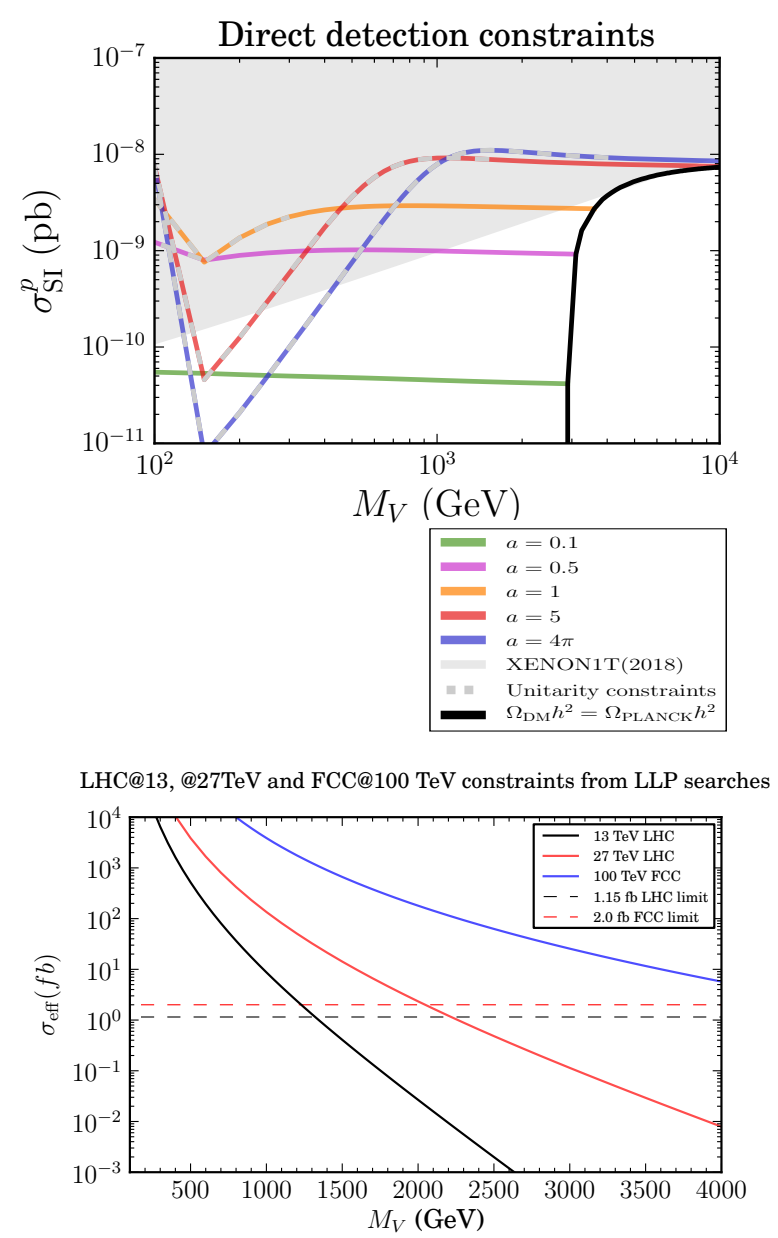

Figure 5: Top: Spin-independent cross-section for $V^{0}$-nucleon elastic scattering as a function of $M_{V}$ and for representative values of $a$. The continuous black curve represents the elastic cross-section computed with the values of $M_{V}$ and $a$ that saturate the measured DM relic density. The grey dashing highlights the parameter space where perturbative unitarity loss occurs at too low scale. Bottom: the effective cross-sections $\sigma_{\text {eff }}=\sigma(p p \rightarrow$ $\left.V^{ \pm} V^{0}\right)+2 \sigma\left(p p \rightarrow V^{+} V^{-}\right)$at leading order for the vector isotriplet model for 13 and $27 \mathrm{TeV}$ LHC energies and for a $100 \mathrm{TeV}$ future collider. The dashed lines corresponds to collider sensitivity. See details in [33].

One should also note that in case of i2HDM, DST signature also allows to substantially enhance LHC potential and probe DM mass upto about $500 \mathrm{GeV}$ [31] which is much higher than $100 \mathrm{GeV}$ - the maximum DM mass which can be probed via mono-jet signature. 


\section{Conclusions}

In the absence of DM signal we can still do a lot - we can prepare ourselves ot its discovery and identification. $\boldsymbol{E}_{\mathrm{T}}^{\text {miss }}$ shape is quite instrumental in understanding the underlying theory at colliders, while direct and indirect DM searches are very powerful in complementing collider searches especially in the parameter space with large DM mass. We also advocate the usage of new DM signatures such as disappearing charge tracks ones which allows to substantially extend collider exploration of large DM mass. Moreover we would like to stress the crucial role of $100 \mathrm{TeV} p p$ collider which is likely to explore the complete parameter space of thermal DM. We show that collider and noncollider DM searches have a unique power to decode the nature of Dark Matter on the examples of several appealing DM theories. Such complementarity and usage of different signatures would allow us to decode the nature of DM, signals from which we are expecting in the near future.

\section{Acknowledgements}

Author would like to thank organisers of EDSU2018 for the invitation and very warm hospitality and acknowledges partial support from the STFC grant ST/L000296/1.

\section{References}

[1] Planck Collaboration, P. A. R. Ade et. al., Astron. Astrophys. 594 (2016) A13 [1502.01589].

[2] G. R. Blumenthal, S. M. Faber, J. R. Primack and M. J. Rees, Nature 311 (1984) 517-525. [,96(1984)].

[3] J. S. Bullock, T. S. Kolatt, Y. Sigad, R. S. Somerville, A. V. Kravtsov, A. A. Klypin, J. R. Primack and A. Dekel, Mon. Not. Roy. Astron. Soc. 321 (2001) 559-575 [astro-ph/9908159].
[4] ATLAS Collaboration, M. Aaboud et. al., JHEP 01 (2018) 126 [1711. 03301$].$

[5] CMS Collaboration, C. Collaboration.

[6] ATLAS Collaboration, Tech. Rep. ATLAS-CONF-2018-005, CERN, Geneva, Apr, 2018.

[7] ATLAS Collaboration, M. Aaboud et. al., Eur. Phys. J. C77 (2017), no. 6393 [1704.03848].

[8] WMAP Collaboration, G. Hinshaw et. al., Astrophys. J. Suppl. 208 (2013) 19 [1212.5226].

[9] M. W. Goodman and E. Witten, Phys. Rev. D31 (1985) 3059. [,325(1984)].

[10] XENON Collaboration, E. Aprile et. al., Phys. Rev. Lett. 119 (2017), no. 18181301 [1705.06655].

[11] LUX Collaboration, D. S. Akerib et. al., Phys. Rev. Lett. 118 (2017), no. 2021303 [1608.07648].

[12] PandaX-II Collaboration, X. Cui et. al., Phys. Rev. Lett. 119 (2017), no. 18181302 [1708.06917].

[13] T. R. Slatyer in Theoretical Advanced Study Institute in Elementary Particle Physics: Anticipating the Next Discoveries in Particle Physics (TASI 2016) Boulder, CO, USA, June 6-July 1, 2016, 2017. 1710.05137.

[14] Fermi-LAT Collaboration, M. Ackermann et. al., Phys. Rev. Lett. 115 (2015), no. 23 231301 [1503.02641].

[15] VERITAS Collaboration, B. Zitzer, $P o S$ ICRC2015 (2016) 1225 [1509. 01105$].$

[16] Fermi-LAT, MAGIC Collaboration, M. L. Ahnen et. al., JCAP 1602 (2016), no. 02039 [1601.06590].

[17] H.E.S.S. Collaboration, H. Abdallah et. al., Phys. Rev. Lett. 117 (2016), no. 11111301 [1607.08142].

[18] H.E.S.S. Collaboration, A. Abramowski et. al., Phys. Rev. Lett. 110 (2013) 041301 [1301.1173]. 
[19] S. Galli, F. Iocco, G. Bertone and A. Melchiorri, Phys. Rev. D80 (2009) 023505 [0905.0003].

[20] S. Galli, F. Iocco, G. Bertone and A. Melchiorri, Phys. Rev. D84 (2011) 027302 [1106.1528].

[21] J. Goodman, M. Ibe, A. Rajaraman, W. Shepherd, T. M. Tait et. al., Phys.Rev. D82 (2010) 116010 [1008.1783].

[22] J. Kumar, D. Marfatia and D. Yaylali, Phys. Rev. D92 (2015), no. 9095027 [1508.04466].

[23] A. Belyaev, L. Panizzi, A. Pukhov and M. Thomas, JHEP 04 (2017) 110 [1610.07545].

[24] A. Belyaev, E. Bertuzzo, C. Caniu Barros, O. Eboli, G. Grilli Di Cortona, F. Iocco and A. Pukhov, 1807.03817.

[25] XENON Collaboration, E. Aprile et. al., Phys. Rev. Lett. 121 (2018), no. 11111302 [1805.12562].
[26] R. J. Hill and M. P. Solon, Phys. Lett. B707 (2012) 539-545 [1111.0016].

[27] M. T. Frandsen, U. Haisch, F. Kahlhoefer, P. Mertsch and K. Schmidt-Hoberg, JCAP 1210 (2012) 033 [1207.3971].

[28] L. Vecchi, 1312.5695.

[29] D. Barducci, A. Belyaev, A. K. M. Bharucha, W. Porod and V. Sanz, JHEP 07 (2015) 066 [1504.02472].

[30] A. Belyaev, T. R. Fernandez Perez Tomei, P. G. Mercadante, C. S. Moon, S. Moretti, S. F. Novaes, L. Panizzi, F. Rojas and M. Thomas, 1809.00933.

[31] A. Belyaev, G. Cacciapaglia, I. P. Ivanov, F. Rojas-Abatte and M. Thomas, Phys. Rev. D97 (2018), no. 3035011 [1612.00511].

[32] R. Mahbubani, P. Schwaller and J. Zurita, JHEP 06 (2017) 119 [1 703.05327 ]. [Erratum: JHEP10,061(2017)].

[33] A. Belyaev, G. Cacciapaglia, J. Mckay, D. Marin and A. R. Zerwekh, 1808.10464. 